\title{
Complementary Methodical Approach for the Analysis of a Perovskite Solar Cell Layered System
}

\author{
Steffi Rades ${ }^{1}$, Frédéric Oswald ${ }^{2}$, Stéphanie Narbey ${ }^{2}$, Jörg Radnik ${ }^{1}$ and Vasile-Dan Hodoroaba ${ }^{1}$ \\ 1. Federal Institute for Materials Research and Testing (BAM), Division Surface Analysis and Interfacial \\ Chemistry, Berlin, Germany \\ 2. Solaronix SA, Aubonne, Switzerland
}

The overall motivation behind this work is to produce thin films perovskite solar cells with constant high light conversion efficiency over time [1,2]. Loss of efficiency may be caused by structural and/or chemical alterations of the complex layered system. As these changes might take place either in the bulk and/or on the surface of the stratified material, analytical tools addressing both key issues are selected and combined. SEM/EDX (on cross-sectioned sample) combined with XPS (for the near-surface region of the unfractured sample) were chosen for development of an appropriate methodical approach to characterize perovskite laboratory cells in depth and complementary on top, before and after light exposure. The layered perovskite system investigated here is based on glass covered with fluorine doped tin oxide (FTO), followed by three porous thin films of $\mathrm{TiO}_{2}, \mathrm{ZrO}_{2}$ and a thick monolithic carbon, see Fig. 1. $\mathrm{TiO}_{2}$ film is subdivided into a dense layer covered by porous one constituted of nanoparticles of truncated bipyramidal shape [3]. This layered system serves as the matrix for the perovskite.

The layered system was sintered at $400^{\circ} \mathrm{C}$ for one hour, then allowed to cool down. Further, perovskite precursor solution consisting of lead iodide and methyl ammonium iodide was infiltrated and samples were annealed at $50^{\circ} \mathrm{C}$ to allow the growth of perovskite crystals. For the SEM/EDX (Zeiss Supra 40 equipped with Bruker XFlash® SDD detector) analyses, the samples have been fractured manually just before the measurements, whereby XPS (AXIS Ultra DLD, Kratos Analytical, UK) analyses were conducted on unfractured samples on a surface area of about $700 \mu \mathrm{m}$ x $300 \mu \mathrm{m}$.

A layout of the solar cell layered system is displayed in Fig. 1 top left. After infiltration of perovskite solution and annealing, EDS spectral maps on cross-sections of specimen have been measured. Distribution of relevant elements $\mathrm{Si}, \mathrm{Sn}, \mathrm{Ti}, \mathrm{Zr}$ and $\mathrm{C}$ correlate conclusively with layers visible in the scheme in Fig.1. Elements $\mathrm{Pb}$ and $\mathrm{I}$ are distributed throughout the porous layers $\mathrm{C}, \mathrm{ZrO}_{2}$ and $\mathrm{TiO}_{2}$. As expected, $\mathrm{Pb}$ and $\mathrm{I}$ are not detected in FTO layer. Specimens were exposed to ambient daylight for 7 weeks. A SEM micrograph of the cross-section of a sample after illumination treatment is shown in Fig. 1 bottom left. Glass substrate and all layers FTO, $\mathrm{TiO}_{2}, \mathrm{ZrO}_{2}$ and $\mathrm{C}$ are clearly identified, as expected. EDX data of samples have been acquired under the same measurement conditions as before the illumination. It was found that several weeks of ambient daylight did not change significantly the qualitative elemental composition nor the distribution of $\mathrm{Pb}$ and $\mathrm{I}$ in the solar cell system (Fig. 1 top right and middle). EDX linescan (intensities normalized to background) in Fig. 1 bottom right suggests a relatively homogeneous distribution of $\mathrm{Pb}$ and $\mathrm{I}$ within $\mathrm{ZrO}_{2}$ and $\mathrm{C}$ layers, and a higher content than in the $\mathrm{TiO}_{2}$ layer. It was confirmed with EDX that nanoparticles identified in high-resolution SEM micrographs contain mainly $\mathrm{Pb}$ and I, indicating these to be the perovskite crystals. However, a timedependent compositional and chemical altering was observed with XPS for the near-surface region of the outermost $\sim 10 \mathrm{~nm}$. In this region, only $\mathrm{C}, \mathrm{O}, \mathrm{N}, \mathrm{I}$ and $\mathrm{Pb}$ were detected with $\mathrm{C}$ as dominating species - as expected. After 2 months of illumination, a significant increase of $\mathrm{I}, \mathrm{Pb}$ and $\mathrm{O}$ was observed. In contrast to this, the fraction of $\mathrm{N}$ decreased. 
From the preliminary results described above it can be inferred that in-depth analysis through the $\sim 10$ $\mu \mathrm{m}$ layered system as well as on its surface by means of the two complementary analytical methods SEM/EDX and XPS is a suitable experimental approach to investigate the photo-electrodes layered system presented here. Systematic time-dependent analyses are ongoing to further investigate the reasons for the loss of efficiency with time. Sources of errors such as matrix and (surface) morphology effects, or stability of the chemistry of the analyzed surface during electron bombardment are evaluated.

\section{References:}

[1] L Etgar et al, J. Am. Chem. Soc. 134 (2012), p. 17396.

[2] J M Ball et al, Energy Environ. Sci. 6 (2013), p. 1739.

[3] E Ortel et al, Microsc. Microanal. 21 (Suppl 3), 2015, p. 2401.

[4] This project has received funding from the European Union's Seventh Framework Programme under grant agreement no. 604577 (SETNanoMetro).

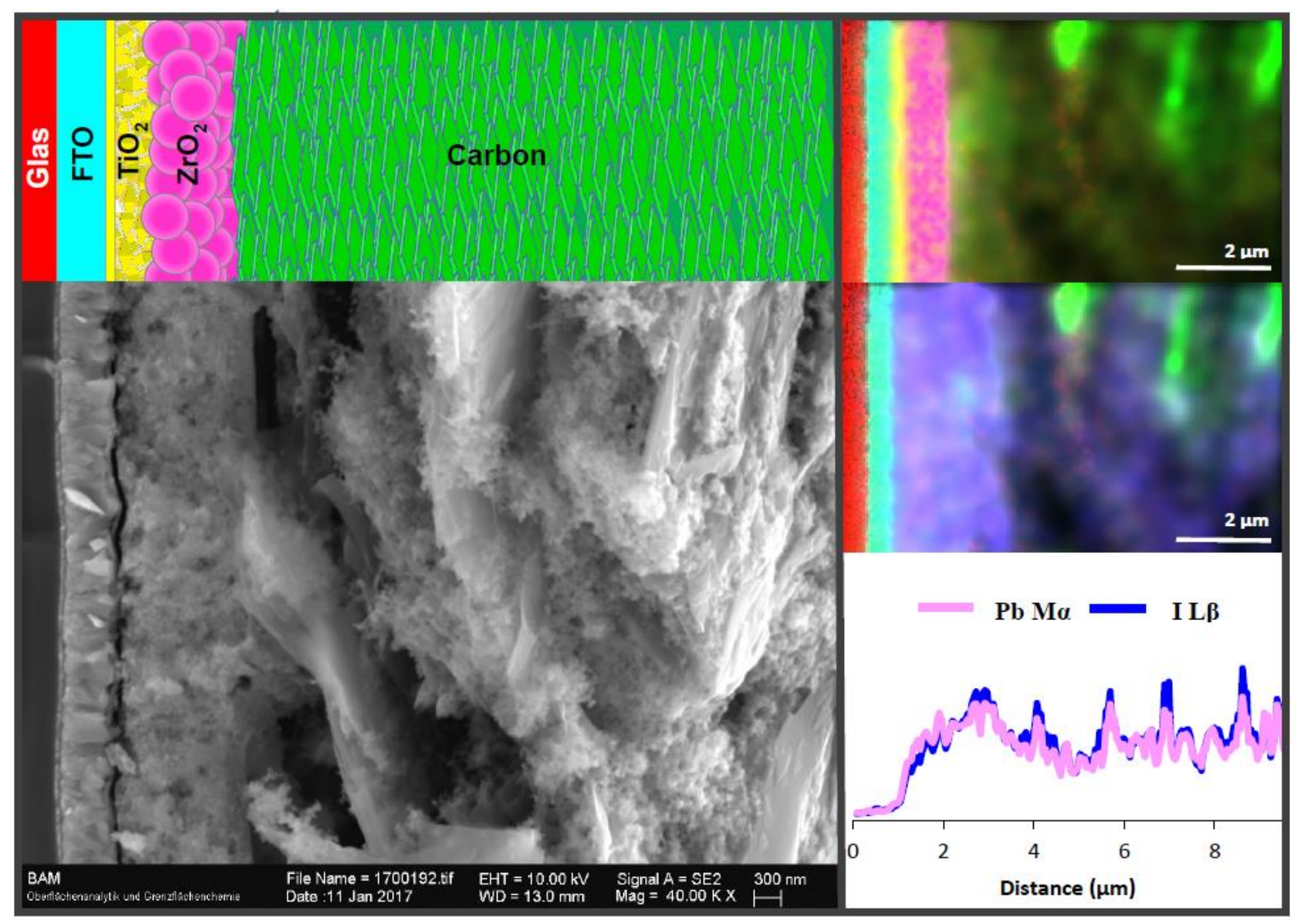

Figure 1. Top left: schematic view of solar cell layered system; bottom left: SEM micrograph of perovskite loaded sample after 7 weeks of ambient light illumination; top and middle right: corresponding EDX spectral images showing elemental distribution of Si (red), Sn (cyan), Ti (yellow), $\mathrm{Zr}$ (magenta) and C (green) and Si (red), Sn (cyan), Pb (rose), I (blue) and C (green), respectively; bottom right: $10 \mathrm{kV}$ EDX linescan of $\mathrm{Pb}$ and I extracted from entire spectral image area above after background normalization (at $2.6 \mathrm{keV}$ ). 